\title{
Posaconazole in the management of refractory invasive fungal infections
}

\author{
Stefan Langner \\ Philipp B Staber \\ Peter Neumeister \\ Division of Hematology, Department \\ of Internal Medicine, Medical \\ University of Graz, Austria
}

\begin{abstract}
The rising incidence of invasive fungal infections due to the expanding population of immunocompromised hosts and the increasing prevalence of fungal resistance has led to the need for novel antifungal agents. Posaconazole, a new member of the triazole class has demonstrated in vitro activity against a broad spectrum of fungi and clinical activity against various fungal pathogens, including Aspergillus spp., Candida spp., zygomycetes, and Fusarium spp. To date, posaconazole has been approved for prophylaxis of invasive fungal infections in stem cell transplant recipients with acute graft versus host disease (GVHD) and neutropenic patients receiving intensive induction chemotherapy for acute myelogenous leukemia and myelodysplastic syndrome. In addition, it has been licensed for use in oropharyngeal candidiasis and for salvage therapy in invasive aspergillosis, fusariosis, coccidioidomycosis, chromoblastomycosis, and mycetoma. Posaconazole is the only azole with activity against zygomycetes and other difficult-to-treat fungi, representing a potential treatment option for refractory invasive mycosis. This article reviews available preclinical and clinical data of posaconazole, focusing on its role in the teatment of refractory invasive fungal infections.
\end{abstract}

Keywords: posaconazole, refractory invasive fungal infections, salvage therapy

\section{Introduction}

Invasive fungal infections (IFI) have increased significantly as a leading cause of life-threatening conditions in immunocompromised patients over the past two decades (Marr et al 2002; Wisplinghoff et al 2004). This is primarily due to the rise of at-risk individuals comprising immuncompromised patients with prolonged neutropenia or advanced HIV infection, and patients who have undergone allogeneic hematopoietic stem cell transplantation (HSCT) or solid organ transplantation. Improvement in anticancer treatment, greater duration and intensity of immunosuppression, and the variety of available antimicrobial therapies have influenced the spectrum of pathogens associated with IFI. Although Candida and Aspergillus spp. remain the principal causes of most IFI, mycoses due to more unusual fungal pathogens like Cryptococcus, Coccidioides, Histoplasma, and agents of zygomycosis (primarily species of Rhizopus, Mucor, Cunninghamella, Apophysomyces, Absidia, and Rhizomucor) have become more prevalent in recent years. In fact, the effectiveness of routine fluconazole prophylaxis has resulted in a reduction of $C$. albicans infections, but has also caused a shift to non-albicans Candida spp., ie, C. krusei and C. glabrata - representing fluconazole-resistant strains - and C. parapsilosis (Pfaller et al 1998; Marr et al 2000; Baran et al 2001). To date only four classes of antifungal agents have been approved for the treatment of IFI: the azoles such as itraconazole, ketokonazole, fluconazole, and voriconazole; the polyenes, the most prominent of which is amphotericin B; flucytosine, a drug inhibiting fungal RNA and DNA synthesis; and the echinocandins, such as caspofungin, anidulafungin, and micafungin (Table 1). Failure rates of these agents, including the recently introduced agents, such as voriconazole and caspofungin, are 


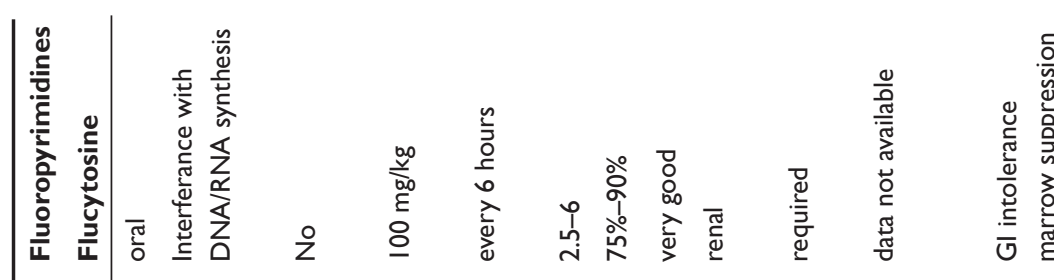

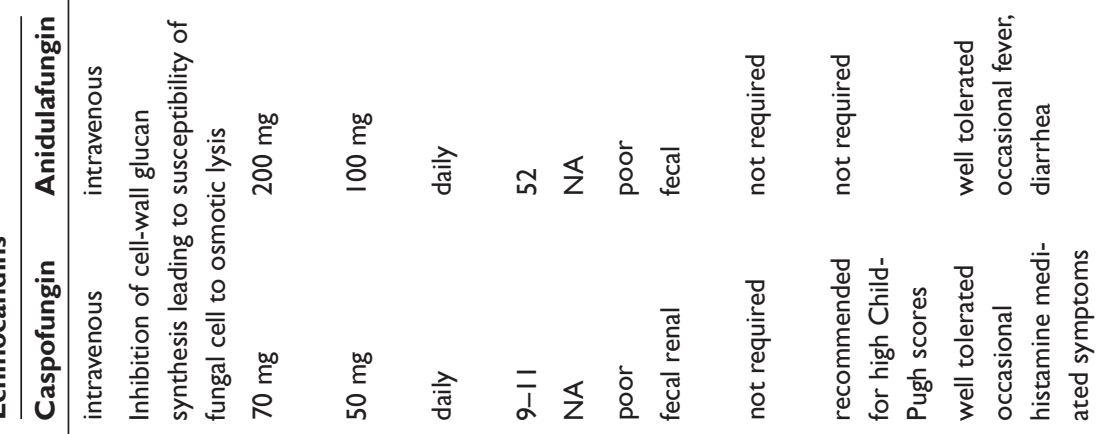

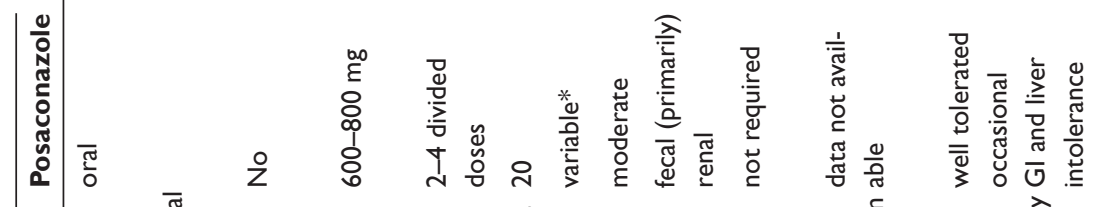

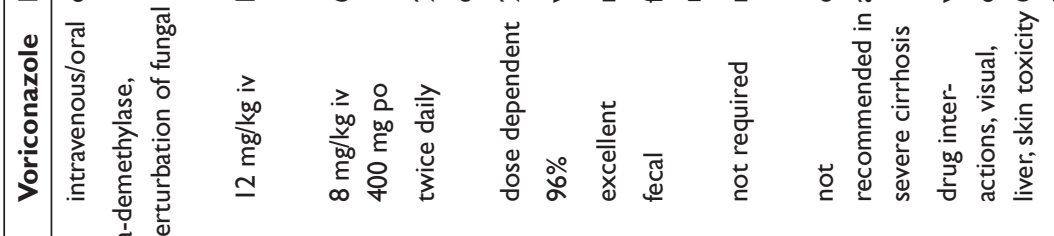

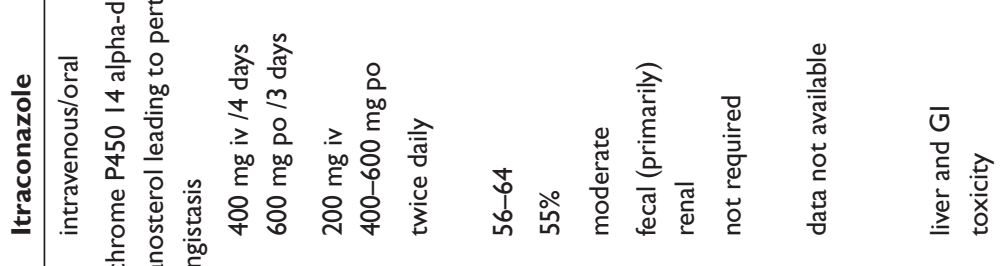

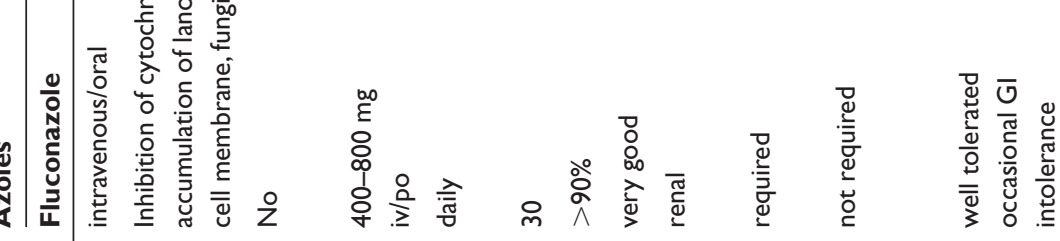

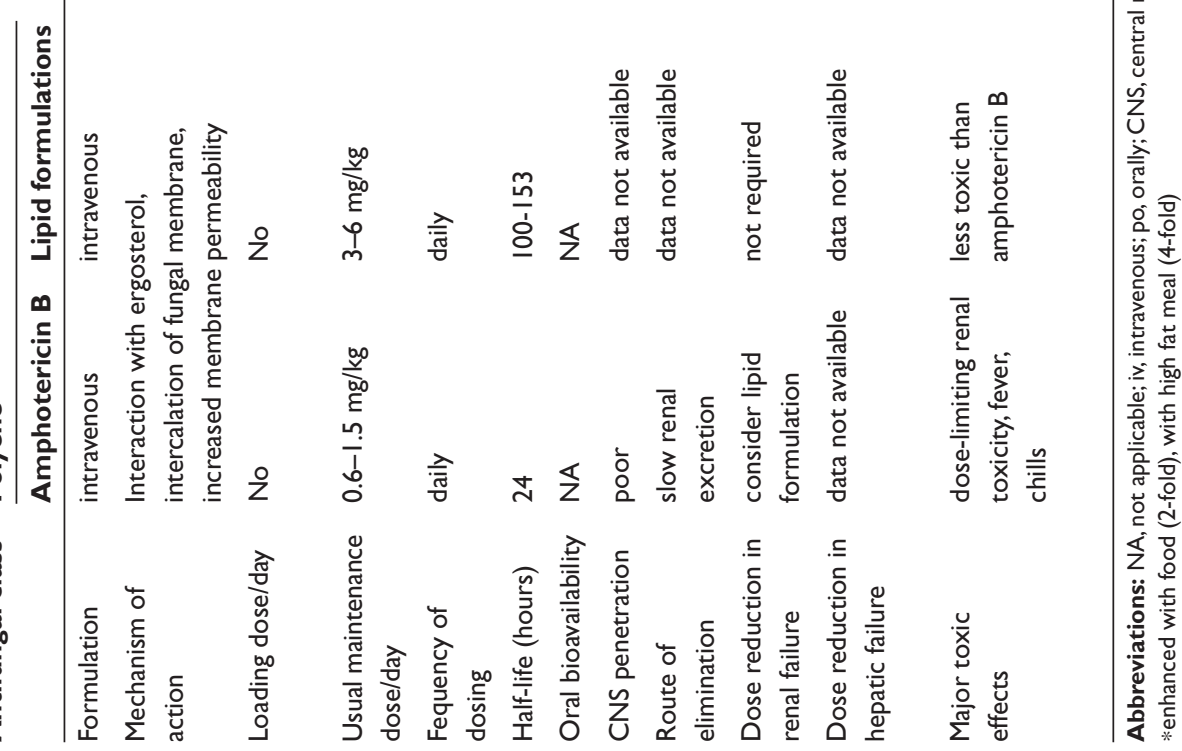


still high - up to $60 \%-80 \%$ in allogeneic HSCT recipients (Barnes and Marr 2007). Hence there is an obvious need for new, more potent, broad-spectrum antifungal agents.

Posaconazole (Noxafil ${ }^{\circledR}$; Schering-Plough), an extendedspectrum triazole has been recently approved for prophylaxis and treatment of refractory IFI by the EMEA and the FDA. The aim of this review is to summarize the in vitro and clinical data available on posaconazole with special focus on refractory invasive mycoses.

\section{Mode of action and mechanism of resistance}

Posaconazole is a lipophilic triazole with structural similarity to itraconazole. The azole class of drugs inhibits the cytochrome P450 (CYP450)-dependent lanosterol 14 alpha-demethylase (CYP51), an enzyme required for ergosterol production which is an essential sterol component in the cell membrane of fungal pathogens but is not present in mammalian cells (Munayyer et al 2004; Wexler et al 2004). Exposure to posaconazole is a more potent inhibitor of sterol synthesis in Aspergillus fumigatus and A. flavus than either itraconazole or voriconazole. Posaconazole has an exceptional high affinity to CYP51. Since posaconazole has a chemical structure different from that of fluconazole and voriconazole, it can interact with an additional domain of the target so that it inhibits even mutated strains resistant to fluconazole and voriconazole (Munayyer et al 2004; Hof 2006). CYP51 is encoded by the ERG11 gene in Candida albicans and is present in almost all yeasts and molds, with the exception of Pneumocystis spp. and Phytium spp. (Hof 2006). Inhibition of CYP51 results in depletion of ergosterol from the fungal cell membrane and accumulation of methylated sterol precursors. This results in membrane instability, increased permeability, and inhibition of cell growth (Kwon et al 2007).

Mechanisms of resistance to azole antifungals have been reported to occur via both mutation of CYP51 and upregulation of genes controlling drug efflux pumps (Lupetti et al 2002; Hof 2006). The target site for the azoles is the ERG11 gene product encoding the cytochrome P450 lanosterol 14 alpha-demethylase, an essential enzyme in the biosynthetic pathway of ergosterol. Many of the identified CYP51 mutations map to the active site of the enzyme, thereby reducing the binding affinity of the respective azoles to their cellular target. Mutations seem to preferentially affect binding of fluconazole and voriconazole versus itraconazole and posaconazole, probably because of the long side chain of the latter two agents (Xiao et al 2004; Kwon and
Mylonakis 2007). Based on 3D models of CYP51 bound to azoles, these side chains occupy a specific channel within CYP51, and this additional interaction serves to stabilize the binding of these azoles to the mutated CYP51 proteins (Xiao et al 2004). The model also predicts that mutations that were previously shown to specifically impact posaconazole susceptibility in A. fumigatus and C. albicans act by interfering with the binding of the long side chain. Azoles passively diffuse into fungal cells and resistance of yeasts as well as molds can develop when transmembrane efflux pumps are activated, thereby decreasing the intracellular drug concentration. The transporters of the ABC family CDR1 and CDR2 together with the major facilitator efflux gene MDR1 have all been implicated in the development of fungal resistance to azoles. However, these efflux pumps seem to work less efficient when exporting posaconazole in comparison to the other azoles (Chau et al 2004; Akins 2005). CDR1 and CDR2 have been shown to efficiently transport fluconazole, ketoconazole, itraconazole, and voriconazole, whereas posaconazole is transported to a much lesser degree. MRD1 can specifically transport fluconazole but has no effect on posaconazole (Akins 2005).

\section{Pharmacokinetics and metabolism}

Posaconazole is available as an oral suspension administered at $800 \mathrm{mg}$ daily in 2 or 4 daily doses for salvage treatment or $600 \mathrm{mg}$ daily in 3 daily doses for prophylaxis. The pharmacokinetics of posaconazole have been studied in single- and multiple-dose studies in healthy volunteers. Collectively, these studies have established that posaconazole displays a linear, dose-proportional pharmacokinetic up to single doses of $800 \mathrm{mg}$ daily (Courtney et al 2003, 2004; Krieter et al 2004). Posaconazole is orally bioavailable, with maximum concentrations reached approximately 10 hours post dose. The absorption of posaconazole is linear through the clinically useful dose of $400 \mathrm{mg}$ every 12 hours; absorption is saturated at higher doses and, thus, loading doses are not possible (Krieter et al 2004). Like itraconazole, food greatly affects posaconazole absorption. Absorption of the oral suspension increases approximately 2-fold when taken with food, and 4-fold with a high-fat meal (Courtney et al 2004). Multiple-dose studies demonstrated that splitting the dose increased the total amount of drug absorbed (Courtney et al 2003). Posaconazole has a large volume of distribution (486 L) - compatible with good tissue penetration - and is $>95 \%$ protein bound. It undergoes limited hepatic metabolism, via UDP glucuronidation and less through the oxidative pathways of the CYP450 system. Elimination 
occurs predominantly through fecal excretion (77\%) and to a lesser extent through urinary excretion (14\%) (Ghosal et al 2004; Krieter et al 2004). As a result of posaconazole's high protein binding and extensive distribution, the terminal plasma elimination half-life is approximately 20 hours, and steady-state concentrations are reached within approximately 7-10 days after initiation of therapy (Krieter et al 2004). This might affect its use in primary therapy for IFI. In a small study comprising patients with varyious degrees of hepatic dysfunction, a trend of increasing half-life indicating prolonged elimination was visible; however, the area under the curve (AUC) did not significantly change and therefore, at this time, no dose adjustment is suggested for patients with hepatic impairment (Courtney et al 2000). In a small study of 24 patients with mild to moderate renal impairment, there was no correlation between pharmacokinetics of posaconazole and mild (creatinine clearance $50-80 \mathrm{~mL} / \mathrm{min}$ ) to moderate (creatinine clearance $20-49 \mathrm{~mL} / \mathrm{min}$ ) renal dysfunction after a single $400 \mathrm{mg}$ oral dose. The drug was not removed by hemodialysis. These data indicate that posaconazole can be administered to subjects with varyious degrees of renal impairment without dose adjustment, and supplemental doses are not needed after hemodialysis (Courtney et al 2005; Ramsewak et al 2005). Mucositis in neutropenic stem cell transplant recipients appears to reduce posaconazole absorption but did not significantly affect mean total posaconazole exposure at steady state condition. Moreover, this reduction could be overcome by increasing the total dose and dosing frequency (Gubbins et al 2006).

\section{Drug interactions}

Drug interactions mediated by various CYP450 are common with the currently available triazole antifungals; however, recent results suggest that posaconazole may have an improved drug interaction profile compared with other triazoles (Wexler et al 2004). Unlike other azoles, posaconazole is not a significant substrate for the CYP450 enzymes and has been shown to inhibit only CYP3A4. Therefore, posaconazole has the potential to interact with other drugs that are metabolized through the 3A4 enzyme system (Ramsewak et al 2005). Pharmacokinetic studies in special populations revealed no necessity for dosage adjustment based on differences in age, gender, race, or renal or hepatic function (Groll and Walsh 2005). The effect of posaconazole on the pharmacokinetics of tacrolimus and ciclosporin was evaluated in healthy subjects and transplant patients revealing that clearance of these immuno-suppressive agents was significantly decreased while the half-life increased. Therefore, when used in combination with mold-active azoles, calcineurin inhibitor doses should be reduced by at least 50\% and their blood or serum concentrations should be closely monitored (Groll and Walsh 2005; Gubbins 2007). The clearance of posaconazole increased 2 -fold in the presence of rifabutin, a potent inducer of CYP3A4. Therefore, co-administration of these two drugs is not advocated. Likewise, concomitant use of posaconazole with phenytoin or cimetidine should be avoided because these drugs decrease posaconazole concentrations by approximately $50 \%$ and $40 \%$, respectively (Torres et al 2005). No dose adjustments are needed when posaconazole is coadministered with drugs such as glipizide, zidovudine, or lamivudine (Groll and Walsh 2005). Co-administration with CYP3A4-metabolised statins (simvastatin, lovastatin, atorvastatin) is contra-indicated as is co-administration with nonsedating antihistamines, cisapride, quinidine, or halofantrine, which may induce QT prolongation and torsades de pointes (Metcalf and Dockrell 2007; Zonios and Bennett 2008). Because interactions caused by itraconazole have been extensively studied and both itraconazole and posaconazole inhibit CYP3A4, drugs with known interaction to itraconazole should be used with caution in posaconazole-treated patients (Zonios and Bennett 2008). Since most drug-drug interaction investigations involving posaonazole have not yet been published, at this time the best sources for potential interactions with posaconazole is the prescribing information.

\section{In vitro studies}

\section{Aspergillus spp./activity against molds}

Filamentous fungal pathogens are increasingly becoming a major cause of IFI in immunocompromised patients. Most of these infections are caused by $A$. fumigatus (90\%), followed by $A$. flavus, A. niger, and A. terreus (Kwon and Mylonakis 2007). Voriconazole is generally regarded as treatment of choice for invasive aspergillosis (Herbrecht et al 2002; Walsh et al 2008). However, Pfaller evaluated the in vitro antifungal activities within the SENTRY Antimicrobial Surveillance Program in the US and Canada of the new triazole antifungal agents, including posaconazole, voriconazole, and itraconazole, as well as amphotericin B, against 239 clinical isolates of filamentous fungi. Overall, posaconazole was the most active compound, inhibiting $94 \%$ of isolates at a minimal inhibitory concentration (MIC) of $\leq 1 \mu \mathrm{g} / \mathrm{mL}$, followed by voriconazole (91\%), amphotericin B (89\%), ravuconazole (88\%), and itraconazole (70\%). Posaconazole also exhibited excellent activity (MICs, 0.03-1 $\mu \mathrm{g} / \mathrm{mL}$ ) 
against less common filamentous fungi tested such as A. niger, A. flavus, A. versicolor, and A. terreus, which is often resistant to amphotericin B. None of the triazoles were active against Fusarium spp. These data have been largely confirmed by a more recent report of the SENTRY study group (Diekema et al 2003). Also, in studies assessing the activity of posaconazole against approximately 19,000 clinically important strains of yeasts and molds including 1,423 Aspergillus spp., isolates collected from 200 medical centers worldwide over a 10-year time span, posaconazole was more or equally active than the comparator drugs itraconazole, fluconazole, voriconazole, and amphotericin B in almost all molds tested. Remarkably, posaconazole was active against isolates of Candida and Aspergillus spp. that exhibited resistance to fluconazole, voriconazole, and amphotericin B and was much more active than the other triazoles against zygomycetes (Sabatelli et al 2006). However, A. niger seems to be less susceptible to posaconazole (Espinel-Ingroff 2003). The clinical implications of raised posaconazole MICs are unclear since it may be possible to achieve clinical serum posaconazole concentrations higher than these MICs (Torres et al 2005).

\section{Zygomycosis}

Zygomycosis is an increasingly emerging life-threatening infection that particularly affects patients with diabetes or malignancy. Posaconazole appears to be the only azole that demonstrates activity against most Zygomycetes. The most common pathogens of human disease are Rhizopus spp. (47\%) and Mucor spp. (18\%), followed by Cunninghamella bertholletiae (7\%) and Apophysomyces elegans (6\%) (Roden et al 2005). Amphotericin B remains first line therapy, but posaconazole has shown in vitro activity against many strains of zygomycetes (Dannaoui et al 2003; Sabatelli et al 2006; Sun et al 2002). A recent comparative study comprising 19,000 yeast and mold isolates, 86 of which were Zygomycetes with voriconazole and itraconazole showed that posaconazole exhibited the lowest MICs against Zygomycetes isolates (Sabatelli et al 2006). In a study of 37 strains of Zygomycetes, posaconazole was effective against Mucor spp., Rhizopus spp., Absidia spp., Rhizomucor spp., C. bertholletiae, and A. elegans (Sun et al 2002; Kwon and Mylonakis 2007).

\section{Candida spp./activity against yeasts}

The incidence of invasive candidiasis is increasing and Candida spp. are now the fourth most common cause of bloodstream infection (Gudlaugsson et al 2003; Pappas 2006).
The mortality attributable to invasive candidiasis ranges from $30 \%$ to $71 \%$ with significant variation between different Candida spp. (ie, in hematologic malignancies the mortality is highest for $C$. albicans and C. tropicalis and lowest for $C$. parapsilosis), as well as underlying conditions, comorbidity and therapeutic procedure (for example HSCT, corticosteroid therapy, antibiotics) (Pagano et al 2006; Staber et al 2007). C. albicans is the most common cause of candidemia in adults and children, and is responsible for $40 \%-77 \%$ of cases (Pappas et al 2006). The in vitro activity of posaconazole has been tested against over 10,000 clinical Candida isolates (Pfaller et al 2004; CuencaEstrella et al 2006; Sabatelli et al 2006). In vitro, posaconazole is highly active against Candida spp. The drug is more active than itraconazole and fluconazole against all Candida spp. and Cryptococcus neoformans. During the ARTEMIS global antifungal surveillance program, 4,169 clinical isolates of Candida spp. were investigated for in vitro susceptibilities of voriconazole, posaconazole, and fluconazole (Pfaller et al 2004). Both voriconazole and posaconazole were more active than fluconazole against all Candida spp. and $C$. neoformans. Posaconazole was the most active azole tested, and was unaffected by fluconazole resistance in $C$. albicans isolates and also had activity against Candida spp. that are commonly azole resistant, including C. glabrata and C. krusei. Posaconazole exhibited fungistatic and fungicidal activity in vitro and in vivo for most Candida spp. isolates and inhibited 97\% of Candida spp. isolates at concentrations of $1 \mu \mathrm{g} / \mathrm{mL}$ or below (Pfaller et al 2001; Espinel-Ingroff 2003). C. albicans is the most susceptible species of Candida whereas C. glabrata and C. pelliculosa are the least susceptible (Pfaller et al 2001, 2004; Carrillo-Munoz et al 2005). Nevertheless, posaconazole showed good activity against fluconazole resistant strains especially Candida glabrata, although it was slightly less active than voriconazole (Sabatelli et al 2006; Pfaller et al 2008).

\section{Cryptococcus neoformans}

Cryptococcosis is one of the leading community-acquired opportunistic mycoses and serious disease (eg, meningitis and cryptococcemia) predominantly occurs in immunocompromised hosts with organ transplantation, hematologic malignancies, or advanced HIV, particularly those not receiving highly active antiretroviral therapy (HAART). Posaconazole has shown good in vitro activity against $C$. neoformans isolates and also inhibited fluconazole-resistant $C$. neoformans. A study of 1,811 global clinical isolates of C. neoformans 
showed that of the 1,646 that were tested against posaconazole, 99\% had MIC $\leq 1 \mu \mathrm{g} / \mathrm{mL}$ (Pfaller et al 2005).

\section{Clinical studies}

Role of posaconazole as salvage therapy for invasive fungal infections

Salvage therapy of IFI generally refers to the treatment of individuals who are refractory or intolerant to initial therapy, administered for at least 1 week. Besides various case reports several clinical trials of salvage therapy have been undertaken, but most were noncomparator studies or contained a nonrandomized control group. Furthermore response criteria used to document refractory disease differed between these studies. Of note, salvage therapy trials frequently include patients both refractory and intolerant to standard therapy, but patients enrolled because of intolerance have a much better response rate (Maertens et al 2004). When evaluating the results from existing salvage studies one has to take these limitations into consideration.

\section{Invasive aspergillosis}

Mortality rates associated with invasive aspergillosis (IA) are still extremely high, particularly in patients undergoing allogeneic HSCT (mortality rate exceeding 50\%) and in patients with central nervous system or disseminated infection (Lin et al 2001; Upton et al 2007). A. fumigatus remains the leading cause for these infections, followed by A. flavus, $A$. niger, and $A$. terreus, a species that is often resistant to antifungal therapy including amphotericin B. At present, voriconazole is recommended as the primary treatment of IA in most patients (Herbrecht et al 2002; Walsh et al 2008). To date only two clinical externally controlled, open label, multi-center trials have investigated posaconazole as salvage therapy in refractory IA. In the first, 107 patients (initially receiving amphotericin $\mathrm{B}$ in most cases) refractory (88\%) or intolerant $(12 \%)$ to conventional therapy were treated with posaconazole ( $800 \mathrm{mg}$ daily in divided doses) for a median duration of 52 days. Selected subjects of the control group received the best available standard of care for salvage therapy. The overall success rate (defined as complete or partial response) was $42 \%$ for posaconzole recipients and $26 \%$ for control subjects (Walsh et al 2007). The second study compared 53 patients with hematologic malignancies receiving posaconazole salvage therapy $(800 \mathrm{mg}$ daily, median duration 70 days) with 52 contemporary controls treated with high-dose lipid formulation of amphotericin B (HD-LPD/AMB at $\geq 7.5 \mathrm{mg} / \mathrm{kg}$ daily) and with 38 other control patients receiving caspofungin and HD-LPD/AMB.
The overall rate of response to posaonazole was $40 \%$, compared with only $8 \%$ and $11 \%$ for HD-LPD/AMB alone or in combination with caspofungin, respectively. After a follow-up of 12 weeks the overall survival was significantly improved in the posaconazole arm (Raad et al 2008).

In conclusion, these studies indicate that posaconazole is an effective option for salvage therapy for invasive aspergillosis predominantly in patients with hematologic malignancies. Nevertheless, randomized clinical trials to define further the role of posaconazole in IA are warranted, including patients with refractory aspergillosis after first-line therapy with voriconazole.

\section{Oropharyngeal candidiasis in patients with HIV}

In patients with HIV and AIDS, oropharyngeal and esophageal candidiasis is the most prevalent opportunistic infection. Fluconazole and itraconazole treatment is usually effective whereas refractory disease can be observed in 5\% of HIV patients, especially in those with advanced HIV infection who have received multiple courses of azole antifungals (Fichtenbaum and Powderly 1998). In the only multi-center, randomized, evaluator-blinded trial of $350 \mathrm{HIV}$ patients with oropharyngeal candidiasis, posaconazole showed noninferiority to fluconazole and a trend to prolonged clinical success after treatment was stopped. Both drugs were administered 14 days (dosed at $200 \mathrm{mg}$ on day one followed by $100 \mathrm{mg}$ daily) and clinical success (evaluated as cure or improvement) was seen in $92 \%$ in the posaconazole versus $95 \%$ in the fluconazole arm (Vazquez et al 2006). Addressing the role of posaconazole in azole-refractory oropharyngeal and esophageal candidiasis, to date two noncomparative, open-label, multicenter studies demonstrated clinical response rates from $73 \%$ to $88 \%$, longterm safety, and good tolerability. Posaconazole was administered $400 \mathrm{mg}$ twice daily, in the first trial (Skiest et al 2007) for a treatment period of 28 days, and up to 12 months in the second trial (Vazquez et al 2007), demonstrating long-term safety, and good tolerability and efficacy.

In summary, posaconazole offers a new, safe, and effective oral treatment option for patients with HIV and azolerefractory mucosal candidiasis.

\section{Zygomycosis}

Zygomycosis is a group of frequently lethal mold infections that usually affects diabetic patients and steroid-treated or severely immunocompromised individuals. Most human infections are caused primarily by species of Rhizopus, Mucor, Cunninghamella, Apophysomyces, Absidia, and 
Rhizomucor. Zygomycetes are highly angioinvasive infections rapidly invading tissue and blood vessels, thereby leading to tissue destruction, thrombosis, infarction, and dissemination. Historically, the agent of choice was conventional amphotericin B at relatively high doses up to 1.5 $\mathrm{mg} / \mathrm{kg}$ daily, which has largely been replaced by less toxic lipid formulations (Walsh et al 1998; Gleissner et al 2004). Two non-comparative, non-randomized open-label studies of posaconazole for salvage therapy of zygomycosis are available. One report analyzed data from 24 subjects refractory or intolerant to predominantly liposomal amphotericin B as first-line therapy. Median time of posaconazole ( $800 \mathrm{mg}$ daily in divided doses) administration was 182 days. Complete response, defined as resolution of signs of infection and no relapse within 30 days after cessation of medication, was observed in $46 \%$, and partial response occurred in 33\%. Five treatment failures occurred, defined as the presence of zygomycosis at the time of termination of posaconazole treatment or at death. Eighteen patients underwent surgical debridement resulting in a significant better survival (Greenberg et al 2006). In a second study, posaconazole (800 mg daily in divided doses) was evaluated for salvage therapy in 91 cases of probable or proven zygomycosis infection. Pretreatment consisted of amphotericin B in most patients. 81 subjects have failed conventional therapy and 10 showed intolerance or both. Overall, at 12 weeks, $60 \%$ of patients had either a complete or partial response to treatment, 17\% demonstrated treatment failure, and the remainder had stable disease. Success rates were similar irrespective of site of infection or whether surgical debridement had been performed (van Burik et al 2006).

In refractory zygomycosis, posaconazole constitutes a suitable oral treatment option although to determine its place as first-line therapy a prospective randomized comparison of posaconazole and lipid amphotericin B needs to be performed.

\section{Other invasive fungal infections Cryptococcal meningitis/fungal CNS infections}

Morbidity and mortality associated with fungal infections of the CNS remains unacceptably high despite treatment with a broad variety of antifungal agents. C. neoformans is the most common fungal agent in patients infected with HIV. Existing treatment guidelines for cryptococcal meningitis recommend therapy with amphotericin B with or without flucytosine (Saag et al 2000); however, clinical response rates in HIV-infected individuals are low. In a multi-center, open-label clinical trial, 39 mostly HIV-positive patients with fungal infection of the CNS (29 of them had cryptococcal meningitis) refractory or intolerant to standard therapy with amphotericin B or fluconazole received posaconazole $800 \mathrm{mg}$ daily for a mean duration of 81 days. Successful outcomes were observed in 14 of 29 (48\%) subjects with cryptococcal meningitis and in 5 of $10(50 \%)$ patients with CNS infections due to other fungal pathogens (Pitisuttithum et al 2005).

These data suggest that posaconazole has clinical activity against fungal infections of the CNS and provides a valuable alternative in patients failing existing antifungal agents.

\section{Coccidioidomycosis}

Coccidioidomycoosis is a systemic fungal infection caused by inhalation of arthroconidia from fungi of the genus Coccidioides. Despite the use of amphotericin B, fluconazole, and itraconazole, disseminated coccidioidomycosis remains difficult to treat, and is characterized by frequent treatment failures and relapses (Galgiani et al 2000). A noncomparative, nonrandomized, multicenter study described 20 patients with chronic pulmonary or nonmeningeal disseminated coccidioidomycosis who received posaconazole (400 mg daily) for up to 6 months. In $85 \%$ of treated individuals a satisfactory response, defined as a $\geq 50 \%$ reduction in the Mycoses Study Group score from baseline, could be documented (Catanzaro et al 2007). In a case series of 6 patients with refractory coccidioidomycosis treated with posaconazole ( $800 \mathrm{mg}$ daily) a successful outcome could be observed in 5 of 6 subjects after 2-34 months of treatment (Anstead et al 2005).

\section{Invasive fusariosis}

Fusarium spp. are among the leading fungal pathogens to cause invasive mold infections in patients with underlying hematopoietic malignancy, particularly in those who have undergone HSCT. Conventional amphotericin B-based therapy for invasive fusariosis in patients with hematologic malignancies resulted in a $>70 \%$ failure rate. Amphotericin B or its lipid formulation represents the treatment of choice, whereas voriconazole is an option for patients refractory or intolerant to first-line therapy. To date one retrospective analysis comprising 21 patients with invasive fusariosis, refractory to or intolerant of standard antifungals (amphotericin B), evaluated posaconazole (800 $\mathrm{mg}$ daily in divided doses) as salvage therapy. After a duration of administration for up to 12 months, an overall response rate of $48 \%$ could be observed, suggesting that posaconazole is useful as an oral treatment for refractory invasive fusariosis (Raad et al 2006b). 


\section{Prophylaxis}

Antifungal prophylaxis with posaconazole can be recommended in HSCT recipients with acute graft versus host disease (GVHD) and in patients during induction chemotherapy for acute myelogenous leukemia or myelodysplastic syndrome. These recommendations are based on two randomized clinical studies. One double-blind trial including 600 patients compared posaconazole $(600 \mathrm{mg}$ daily in divided doses) with fluconazole (400 mg daily) as prophylaxis against IFI in allogeneic HSCT recipients receiving immunosuppressive therapy for treatment of GVHD. At 4 months, posaconazole was found to be as effective as fluconazole in preventing all IFI (incidence: $5.3 \%$ vs $9.0 \%, p=0.07$ ) and was superior to fluconazole in preventing proven or probable invasive aspergillosis $(2.3 \%$ vs $7.0 \%, p=0.006)$. Overall mortality was similar in the two groups, but deaths from IFI were lower in the posaconazole group (Ullmann et al 2007). The second study of 602 subjects compared posaconazole (600 mg daily in divided doses) with fluconazole (400 mg daily) or itraconazole (200 $\mathrm{mg}$ twice daily) as prophylaxis for IFI in neutropenic patients receiving chemotherapy for acute myelogenous leukemia or myelodysplastic syndrome (Cornely et al 2007). At 3 months posaconazole was found to be superior to the combined fluconazole/itraconazole group in preventing IFI $(2 \%$ vs $8 \%, p<0.001)$. Rates of invasive aspergillosis ( $1 \%$ vs $7 \%, \mathrm{p}<0.001)$ were also less with posaconazole and a significantly improved survival could be observed $(p=0.04)$. Posaconazole treatment was relatively well tolerated in both studies.

\section{Safety and tolerability}

During phase I, II, and III clinical trials, posaconazole was demonstrated to be safe and well tolerated. In phase I studies most adverse events were mild, transient, and nonspecific, the most common adverse events including gastrointestinal disturbances, headache, dry mouth, somnolence, dizziness, fatigue, and constipation (Courtney et al 2003; Ezzet et al 2005). In a study of 428 patients with refractory invasive fungal infections or febrile neutropenia, 109 of whom continued treatment for $\geq 6$ months, $38 \%$ reported adverse reactions (Raad et al 2006a). However, most of these were mild, nausea being the most common (8\%), followed by vomiting (6\%) and abdominal pain (4\%). QT interval prolongation was observed in $1 \%$ of patients and elevation of liver enzymes in $2 \%$. Adverse events occurred at similar rates in patients who received posaconazole therapy for $<6$ months or those treated for $<6$ months. Long-term therapy did not increase the risk of any individual adverse event, and no unique adverse event was observed with longer exposure to posaconazole (Raad et al 2006a). In two large controlled trials, posaconazole seems to have a comparable safety profile as fluconazole or itraconazole. In a study comprising more than 600 patients comparing posaconazole vs fluconazole or itraconazole prophylaxis in patients with neutropenia, serious adverse events possibly or probably related to treatment were reported by $6 \%$ in the posaconazole group and in $2 \%$ in the fluconazole or itraconazole group. The most common treatment-related adverse events in both groups were gastrointestinal tract disturbances (Cornely et al 2007; Ullmann et al 2007). Posaconazole therapy is also safe and well tolerated in pediatric and elderly patients and treatment-related discontinuations are uncommon (Torres et al 2005).

\section{Conclusions and place in therapy}

Invasive fungal infections cause substantial morbidity and mortality in immunocompromised patients. Since early detection and early effective treatment of invasive fungal infections can be life saving, the most common approach is antifungal prophylaxis and empirical therapy in neutropenic patients with persistent fever. For the future, a more refined approach such as pre-emptive therapy - only initiated upon identification of fungal markers in combination with clinical and radiologic signs - may improve the definition of target patients and expose fewer patients to potentially toxic and/or expensive treatment. The response rate to therapy, in particular for invasive aspergillosis and other invasive mold infections, has been poor. With the advance of a number of techniques facilitating early diagnosis together with the development of new antifungals like the echinocandins or extended spectrum azols like voriconazole or posaconazole the outcomes for invasive fungal infection in immunocompromised patients could be improved. In this context, caspofungin has emerged as primary therapy of invasive candidiasis (Mora-Duarte et al 2002) and neutropenic fever (Walsh et al 2004) and voriconacole has largely replaced amphotericin B as first line therapy of invasive aspergillosis (Herbrecht et al 2002). Posaconazole is the most broad-spectrum azole antifungal to date, with activity against Candida spp., including fluconazole-resistant isolates, and Aspergillus spp. Posaconazole is likely to be an important new agent in the antifungal armamentarium. The primary role for posaconazole will be the prophylaxis of IFI in severely immunocompromised patients such as HSCT recipients with acute GVHD (Ullmann et al 2007) and neutropenic patients receiving 
intensive induction chemotherapy for acute myelogenous leukaemia and myelodysplastic syndrome (Cornely et al 2007). The European Conference on Infections in Leukemia (ECIL) guidelines therefore included a provisional AI (ie, strongly recommended, based on at least one well-executed, randomized trial) recommendation for these indications (Maertens 2007). However, the prophylactic use of such a broad spectrum agent may lead to the emergence of fungal breakthrough infections as it has been suggested that use of voriconazole as fungal prophylaxis has led to a possible increase of zygomycosis infections in immunocompromised patients. Posaconazole is also approved as first-line treatment of oropharyngeal candidasis including those refractory to fluconazole or itraconazole in HIV positive patients. There is already clinical evidence for its efficacy as salvage therapy in a number of invasive fungal infections. Posaconazole is the only azole to have activity against the zygomycetes, including Mucor, Rhizopus, and other species, and has shown clinical effectiveness against refractory infections caused by these fungi. In addition, there are reasonable data showing posaconazole exerts activity against rare, difficult-to-treat fungi such as Fusarium and Scedosporium spp., and has also activity against $C$. neoformans. Similarly, preliminary evidence from noncomparative salvage trials suggests its efficacy as second-line therapy for invasive Aspergillus infection. Nevertheless, many therapeutic areas of uncertainty remain. Amphotericin B and caspofungin remain the only approved agents for the antifungal management of febrile neutropenia, but the broad spectrum of posaconazole makes it an attractive target for further clinical studies. In addition, its role in the treatment of Candida spp. remains to be established since no randomized controlled studies in yeast infections are available. Finally, the role of combination therapies of posaconazole with classes of drugs targeting different pathways against filamentous fungi (ie, echinocandins) seems to be a promising task and will provide a focus for future studies.

\section{Disclosures}

None of the authors has any conflicts of interest to disclose.

\section{References}

Akins RA. 2005. An update on antifungal targets and mechanisms of resistance in Candida albicans. Med Mycol, 43:285-318.

Anstead GM, Corcoran G, Lewis J, et al. 2005. Refractory coccidioidomycosis treated with posaconazole. Clin Infect Dis, 40:1770-6.

Baran J, Jr., Muckatira B, Khatib R. 2001. Candidemia before and during the fluconazole era: prevalence, type of species and approach to treatment in a tertiary care community hospital. Scand J Infect Dis, 33:137-9.
Barnes PD, Marr KA. 2007. Risks, diagnosis and outcomes of invasive fungal infections in haematopoietic stem cell transplant recipients. Br J Haematol, 139:519-31.

Carrillo-Munoz AJ, Quindos G, Ruesga M, et al. 2005. Antifungal activity of posaconazole compared with fluconazole and amphotericin B against yeasts from oropharyngeal candidiasis and other infections. J Antimicrob Chemother, 55:317-19.

Catanzaro A, Cloud GA, Stevens DA, et al. 2007. Safety, tolerance, and efficacy of posaconazole therapy in patients with nonmeningeal disseminated or chronic pulmonary coccidioidomycosis. Clin Infect Dis, 45:562-8.

Chau AS, Mendrick CA, Sabatelli FJ, et al. 2004. Application of realtime quantitative PCR to molecular analysis of Candida albicans strains exhibiting reduced susceptibility to azoles. Antimicrob Agents Chemother, 48:2124-31.

Cornely OA, Maertens J, Winston DJ, et al. 2007. Posaconazole vs. fluconazole or itraconazole prophylaxis in patients with neutropenia. $N$ Engl J Med, 356:348-59.

Courtney RD, Laughlin M, Gontz H, et al. 2000. Single-dose pharmacokinetics of posaconazole in subjects with various degrees of chronic liver disease [abstract]. AAPS PharmSci, AAPS Annual Meeting Supplement. Abstract 3495 .

Courtney R, Pai S, Laughlin M, et al. 2003. Pharmacokinetics, safety, and tolerability of oral posaconazole administered in single and multiple doses in healthy adults. Antimicrob Agents Chemother, 47:2788-95.

Courtney R, Radwanski E, Lim J, et al. 2004. Pharmacokinetics of posaconazole coadministered with antacid in fasting or nonfasting healthy men. Antimicrob Agents Chemother, 48:804-8.

Courtney R, Sansone A, Smith W, et al. 2005. Posaconazole pharmacokinetics, safety, and tolerability in subjects with varying degrees of chronic renal disease. J Clin Pharmacol, 45:185-92.

Cuenca-Estrella M, Gomez-Lopez A, Mellado E, et al. 2006. Head-to-head comparison of the activities of currently available antifungal agents against 3,378 Spanish clinical isolates of yeasts and filamentous fungi. Antimicrob Agents Chemother, 50:917-21.

Dannaoui E, Meletiadis J, Mouton JW, et al. 2003. In vitro susceptibilities of zygomycetes to conventional and new antifungals. $J$ Antimicrob Chemother, 51:45-52.

Diekema DJ, Messer SA, Hollis RJ, et al. 2003. Activities of caspofungin, itraconazole, posaconazole, ravuconazole, voriconazole, and amphotericin B against 448 recent clinical isolates of filamentous fungi. J Clin Microbiol, 41:3623-6.

Espinel-Ingroff A. 2003. In vitro antifungal activities of anidulafungin and micafungin, licensed agents and the investigational triazole posaconazole as determined by NCCLS methods for 12,052 fungal isolates: review of the literature. Rev Iberoam Micol, 20:121-36.

Ezzet F, Wexler D, Courtney R, et al. 2005. Oral bioavailability of posaconazole in fasted healthy subjects: comparison between three regimens and basis for clinical dosage recommendations. Clin Pharmacokinet, 44:211-20.

Fichtenbaum CJ, Powderly WG. 1998. Refractory mucosal candidiasis in patients with human immunodeficiency virus infection. Clin Infect Dis, 26:556-65.

Galgiani JN, Catanzaro A, Cloud GA, et al. 2000. Comparison of oral fluconazole and itraconazole for progressive, nonmeningeal coccidioidomycosis. A randomized, double-blind trial. Mycoses Study Group. Ann Intern Med, 133:676-86.

Ghosal A, Hapangama N, Yuan Y, et al. 2004. Identification of human UDPglucuronosyltransferase enzyme(s) responsible for the glucuronidation of posaconazole (Noxafil). Drug Metab Dispos, 32:267-71.

Gleissner B, Schilling A, Anagnostopolous I, et al. 2004. Improved outcome of zygomycosis in patients with hematological diseases? Leuk Lymphoma, 45:1351-60.

Greenberg RN, Mullane K, van Burik JA, et al. 2006. Posaconazole as salvage therapy for zygomycosis. Antimicrob Agents Chemother, 50:126-33.

Groll AH, Walsh TJ. 2005. Posaconazole: clinical pharmacology and potential for management of fungal infections. Expert Rev Anti Infect Ther, 3:467-87. 
Gubbins PO. 2007. Mould-active azoles: pharmacokinetics, drug interactions in neutropenic patients. Curr Opin Infect Dis, 20:579-86.

Gubbins PO, Krishna G, Sansone-Parsons A, et al. 2006. Pharmacokinetics and safety of oral posaconazole in neutropenic stem cell transplant recipients. Antimicrob Agents Chemother, 50:1993-9.

Gudlaugsson O, Gillespie S, Lee K, et al. 2003. Attributable mortality of nosocomial candidemia, revisited. Clin Infect Dis, 37:1172-7.

Herbrecht R, Denning DW, Patterson TF, et al. 2002. Voriconazole versus amphotericin B for primary therapy of invasive aspergillosis. $N$ Engl $J$ Med, 347:408-15.

Hof H. 2006. A new, broad-spectrum azole antifungal: posaconazolemechanisms of action and resistance, spectrum of activity. Mycoses, 49(Suppl 1):2-6.

Krieter P, Flannery B, Musick T, et al. 2004. Disposition of posaconazole following single-dose oral administration in healthy subjects. Antimicrob Agents Chemother, 48:3543-51.

Kwon DS, Mylonakis E. 2007. Posaconazole: a new broad-spectrum antifungal agent. Expert Opin Pharmacother, 8:1167-78.

Lin SJ, Schranz J, Teutsch SM. 2001. Aspergillosis case-fatality rate: systematic review of the literature. Clin Infect Dis, 32:358-66.

Lupetti A, Danesi R, Campa M, et al. 2002. Molecular basis of resistance to azole antifungals. Trends $\mathrm{Mol} \mathrm{Med,} \mathrm{8:76-81.}$

Maertens J. 2007. Evaluating prophylaxis of invasive fungal infections in patients with haematologic malignancies. Eur J Haematol, 78:275-82.

Maertens J, Raad I, Petrikkos G, et al. 2004. Efficacy and safety of caspofungin for treatment of invasive aspergillosis in patients refractory to or intolerant of conventional antifungal therapy. Clin Infect Dis, 39:1563-71.

Marr KA, Carter RA, Crippa F, et al. 2002. Epidemiology and outcome of mould infections in hematopoietic stem cell transplant recipients. Clin Infect Dis, 34:909-17.

Marr KA, Seidel K, White TC, et al. 2000. Candidemia in allogeneic blood and marrow transplant recipients: evolution of risk factors after the adoption of prophylactic fluconazole. J Infect Dis, 181:309-16.

Metcalf SC, Dockrell DH. 2007. Improved outcomes associated with advances in therapy for invasive fungal infections in immunocompromised hosts. J Infect, 55:287-99.

Mora-Duarte J, Betts R, Rotstein C, et al. 2002. Comparison of caspofungin and amphotericin B for invasive candidiasis. $N$ Engl J Med, 347:2020-9.

Munayyer HK, Mann PA, Chau AS, et al. 2004. Posaconazole is a potent inhibitor of sterol 14alpha-demethylation in yeasts and molds. Antimicrob Agents Chemother, 48:3690-6.

Pagano L, Caira M, Candoni A, et al. 2006. The epidemiology of fungal infections in patients with hematologic malignancies: the SEIFEM-2004 study. Haematologica, 91:1068-75.

Pappas PG. 2006. Invasive candidiasis. Infect Dis Clin North Am, 20:485-506.

Pappas PG, Rex JH, Lee J, et al. 2003. A prospective observational study of candidemia: epidemiology, therapy, and influences on mortality in hospitalized adult and pediatric patients. Clin Infect Dis, 37:634-43.

Pfaller MA, Jones RN, Messer SA, et al. 1998. National surveillance of nosocomial blood stream infection due to Candida albicans: frequency of occurrence and antifungal susceptibility in the SCOPE Program. Diagn Microbiol Infect Dis, 31:327-32.

Pfaller MA, Messer SA, Boyken L, et al. 2004. In vitro activities of voriconazole, posaconazole, and fluconazole against 4,169 clinical isolates of Candida spp. and Cryptococcus neoformans collected during 2001 and 2002 in the ARTEMIS global antifungal surveillance program. Diagn Microbiol Infect Dis, 48:201-5.

Pfaller MA, Messer SA, Boyken L, et al. 2005. Global trends in the antifungal susceptibility of Cryptococcus neoformans (1990 to 2004). $J$ Clin Microbiol, 43:2163-7.

Pfaller MA, Messer SA, Hollis RJ, et al. 2001. In vitro activities of posaconazole (Sch 56592) compared with those of itraconazole and fluconazole against 3,685 clinical isolates of Candida spp. and Cryptococcus neoformans. Antimicrob Agents Chemother, 45:2862-4.
Pfaller MA, Messer SA, Boyken L, et al. 2008. Selection of a surrogate agent (fluconazole or voriconazole) for initial susceptibility testing of posaconazole against Candida spp.: results from a global antifungal surveillance program. J Clin Microbiol, 46:551-9.

Pitisuttithum P, Negroni R, Graybill JR, et al. 2005. Activity of posaconazole in the treatment of central nervous system fungal infections. J Antimicrob Chemother, 56:745-55.

Raad II, Graybill JR, Bustamante AB, et al. 2006a. Safety of long-term oral posaconazole use in the treatment of refractory invasive fungal infections. Clin Infect Dis, 42:1726-34.

Raad, II, Hachem RY, Herbrecht R, et al. 2006b. Posaconazole as salvage treatment for invasive fusariosis in patients with underlying hematologic malignancy and other conditions. Clin Infect Dis, 42:1398-403.

Raad, II, Hanna HA, Boktour M, et al. 2008. Novel antifungal agents as salvage therapy for invasive aspergillosis in patients with hematologic malignancies: posaconazole compared with high-dose lipid formulations of amphotericin B alone or in combination with caspofungin. Leukemia, 22:496-503.

Ramsewak SF, Kuti JL, Nicolau DP. 2005. Focus on posaconazole: A novel triazole antifungal for the treatment of invasive fungal infections. Formulary, 40:13-21.

Roden MM, Zaoutis TE, Buchanan WL, et al. 2005. Epidemiology and outcome of zygomycosis: a review of 929 reported cases. Clin Infect Dis, 41:634-53.

Saag MS, Graybill RJ, Larsen RA, et al. 2000. Practice guidelines for the management of cryptococcal disease. Infectious Diseases Society of America. Clin Infect Dis, 30:710-18.

Sabatelli F, Patel R, Mann PA, et al. 2006. In vitro activities of posaconazole, fluconazole, itraconazole, voriconazole, and amphotericin B against a large collection of clinically important molds and yeasts. Antimicrob Agents Chemother, 50:2009-15.

Skiest DJ, Vazquez JA, Anstead GM, et al. 2007. Posaconazole for the treatment of azole-refractory oropharyngeal and esophageal candidiasis in subjects with HIV infection. Clin Infect Dis, 44:607-14.

Staber P, Langner S, Dornbusch HJ, et al. 2007. Antifungal management in cancer patients. Wien Med Wochenschr, 157:503-10.

Sun QN, Fothergill AW, McCarthy DI, et al. 2002. In vitro activities of posaconazole, itraconazole, voriconazole, amphotericin B, and fluconazole against 37 clinical isolates of zygomycetes. Antimicrob Agents Chemother, 46:1581-2.

Torres HA, Hachem RY, Chemaly RF, et al. 2005. Posaconazole: a broadspectrum triazole antifungal. Lancet Infect Dis, 5:775-85.

Ullmann AJ, Lipton JH, Vesole DH, et al. 2007. Posaconazole or fluconazole for prophylaxis in severe graft-versus-host disease. $N$ Engl J Med, 356:335-47.

Upton A, Kirby KA, Carpenter P, et al. 2007. Invasive aspergillosis following hematopoietic cell transplantation: outcomes and prognostic factors associated with mortality. Clin Infect Dis, 44:531-40.

van Burik JA, Hare RS, Solomon HF, et al. 2006. Posaconazole is effective as salvage therapy in zygomycosis: a retrospective summary of 91 cases. Clin Infect Dis, 42:e61-65.

Vazquez JA, Skiest DJ, Nieto L, et al. 2006. A multicenter randomized trial evaluating posaconazole versus fluconazole for the treatment of oropharyngeal candidiasis in subjects with HIV/AIDS. Clin Infect Dis, 42:1179-86.

Vazquez JA, Skiest DJ, Tissot-Dupont H, et al. 2007. Safety and efficacy of posaconazole in the long-term treatment of azole-refractory oropharyngeal and esophageal candidiasis in patients with HIV infection. HIV Clin Trials, 8:86-97.

Walsh TJ, Anaissie EJ, Denning DW, et al. 2008. Treatment of aspergillosis: clinical practice guidelines of the Infectious Diseases Society of America. Clin Infect Dis, 46:327-60.

Walsh TJ, Hiemenz JW, Seibel NL, et al. 1998. Amphotericin B lipid complex for invasive fungal infections: analysis of safety and efficacy in 556 cases. Clin Infect Dis, 26:1383-96.

Walsh TJ, Raad I, Patterson TF, et al. 2007. Treatment of invasive aspergillosis with posaconazole in patients who are refractory to or intolerant of conventional therapy: an externally controlled trial. Clin Infect Dis, 44:2-12. 
Walsh TJ, Teppler H, Donowitz GR, et al. 2004. Caspofungin versus liposomal amphotericin B for empirical antifungal therapy in patients with persistent fever and neutropenia. $N$ Engl J Med, 351:1391-402.

Wexler D, Courtney R, Richards W, et al. 2004. Effect of posaconazole on cytochrome P450 enzymes: a randomized, open-label, two-way crossover study. Eur J Pharm Sci, 21:645-53.

Wisplinghoff H, Bischoff T, Tallent SM, et al. 2004. Nosocomial bloodstream infections in US hospitals: analysis of 24,179 cases from a prospective nationwide surveillance study. Clin Infect Dis, 39:309-17.
Xiao L, Madison V, Chau AS, et al. 2004. Three-dimensional models of wildtype and mutated forms of cytochrome P450 14alpha-sterol demethylases from Aspergillus fumigatus and Candida albicans provide insights into posaconazole binding. Antimicrob Agents Chemother, 48:568-74.

Zonios DI, Bennett JE. 2008. Update on azole antifungals. Semin Respir Crit Care Med, 29:198-210. 
